\title{
Congenital duodenal obstruction: Ten-year results of a
}

\section{tertiary center}

\author{
Veli Avci ${ }^{*}$, Salim Bilici ${ }^{2}$, Ebuzer Düz ${ }^{1}$, Burhan Beger ${ }^{1}$, Ibrahim Değer ${ }^{3}$ \\ ${ }^{1}$ Department of Pediatric Surgery, Yuzuncu Yil University, Medical Faculty, Van, Turkey \\ ${ }^{2}$ Health Sciences University Diyarbakir Gazi Yasargil Training and Research Hospital Clinic of Pediatric Surgery \\ Diyarbaker, Turkey \\ ${ }^{3}$ Department of Pediatrics, Division of Neonatology, Yuzuncu Yil University, Medical Faculty, V an, Turkey
}

\begin{abstract}
The most common place of gastrointestinal obstruction in the newborn is duodenum. In this study, we aimed to present our 10 years of experience about patients that diagnosed with congenital duodenal obstruction by the guidence of literature.

Between 2008 and 2017, patients who underwent surgery for congenital duodenal obstruction were evaluated retrospectively in terms of age, gender, birth weight, symptom-findings, additional congenital anomalies, treatment modalities, postoperative complications, average length of hospitalization and morbidity-mortality.

A total of 32 patients (18 male, 14 female) with congenital duodenal obstruction were included in the study. The mean birth weight of the patients was $1920 \pm 1130$ grams. Vomiting $(75 \%)$ was the most common symptom. Surgical intervention was performed with an average of two days; the most prefered $(59 \%)$ method was duodenodeudenostomy. The most common complication after surgery was wound infections $(22 \%) .50 \%$ of the patients had additional congenital anomalies. Down syndrome was the most common one. Two patients died due to sepsis. The average length of hospitalization was $14 \pm 5$ days.

For a good outcome in congenital duodenal obstruction, the tips of early diagnosis and treatment should be known.
\end{abstract}

Key Words: Newborn, duodenum, obstruction, treatment

\section{Introduction}

Although the lack of recanalization of the duodenal lumen is blamed, pathogenesis of the congenital duodenal obstruction (CDO) still remains unclear $(1,2)$. In the 1931, the first operation of CDO was performed by Ladd. In that time mortality rate was reported about $40 \%$ but it has been reduced to $5 \%$ in recent years due to improvements in neonatal intensive care and pediatric anesthesia (3). Published studies on duodenal obstruction are limited in Turkey. As the publications in this subject increase, the characteristics of the disease will also emerge. With this study, we aimed to contribute to the literature with the analysis and documentation of our 10 years of experiences.

\section{Material and Methods}

This study was carried out according to the principles of the Helsinki Declaration. In addition, the study was approved by the local ethics committee dated 31.01.2018 and number 01.Patients who underwent surgery between January 2008 and December 2017 were evaluated retrospectively in terms of age, gender, birth weight, symptom-findings, prematurity, age of diagnosis, time for oral feeding, additional congenital anomalies, diagnosis and treatment methods, length of hospitalization, postoperative complications and morbidity-mortality. Patients in the study were divided into two groups according to CDO Ladd Classification; intrinsic (atresia, stenosis and web) and extrinsic (annular pancreas, Ladd's band, Superior Mesenteric Artery pressure and rarely preduodenal portal vein) factors. These two groups were compared according to documentation.

Statistical Analysis: Descriptive statistics for the continuous variables (characteristics) were presented as mean, standard deviation while count and percent for the categorical variables. The SPSS 13.0 for Windows (SPSS Inc. Chicago, IL, USA) statistical program was used for all statistical computations.

\section{Result}

Out of 32 patients who were diagnosed with CDO, 18 $(56 \%)$ were male and $14(44 \%)$ were female. The mean birth weight of the patients was $1920 \pm 1130$ grams. $14(44 \%)$ of the patients were premature. Among the symptoms, vomiting was most common with $75 \%(\mathrm{n}=24)$. Epigastric fullness was another symptom that is less common ( $\mathrm{n}=12,38 \%$ ). 16 patients $(50 \%)$ had additional congenital anomalies; Down syndrome was the most common one (Table 1). $22(69 \%)$ of the patients were diagnosed by the first day of life. 
Table 1. Additional congenital anomalies

\begin{tabular}{lcc}
\hline & Number of Patients & Percent of Patients \\
\hline Down syndrome & 11 & 69 \\
Congenital heart disease & 2 & 13 \\
Esophagial atresia & 1 & 6 \\
Urinary system malformation & 1 & 6 \\
Anorectal anomalies & 1 & 6 \\
\hline Total & 16 & 100 \\
\hline
\end{tabular}

Table 2. Surgical methods of congenital duodenal obstruction

\begin{tabular}{lcc}
\hline & Number of Patients & Percent of Patients \\
\hline Duodenodeudenostomy & 19 & 59 \\
Duodenojejunostomy & 6 & 19 \\
Ladd's band excision & 4 & 13 \\
Web excision with duodenotomy & 2 & 6 \\
Gastroduodenostomy & 1 & 3 \\
\hline Total & 32 & 100 \\
\hline
\end{tabular}

Surgical intervention was performed approximately two days after birth. A double-bubble image was obtained on flat and erect abdominal $\mathrm{x}$ ray in 24 $(75 \%)$ patients. Five $(16 \%)$ patients were diagnosed incidentally after the orogastric catheter was advanced to the distal canal during acute abdominal surgery and in three $(9 \%)$ patients diagnose were put by contrasted abdominal $\mathrm{x}$-ray. Some patients $(\mathrm{n}=12$, $38 \%$ ) were diagnosed during intrauterine period. Entire CDO diagnosed patients in the intrauterine period, were checked after birth again and a doublebubble image was detected on the flat and erect abdominal $x$ ray. Patients are operated after fluidelectrolyte therapy and antibiotic prophylaxis. Duodenodeudenostomy was the most common procedure $(\mathrm{n}=19,59 \%)$ (Table 2$)$. In the early postoperative period, wound infections developed in seven of the patients $(22 \%)$. In the late post-discharge period, two patients $(6 \%)$ required re-surgery due to intraabdominal adhesions. Nutrition was initiated three days after the surgery with the help of an oroduodenal catheter. The duodenal catheter was withdrawn on the sixth day. Atresia $(n=10,31 \%)$ from intrinsic reasons and annular pancreas $(n=9$, $28 \%$ ) from extrinsic reasons were the most common reasons of obstruction in the study (Table 3).

18 patients had intrinsic causes and 14 had extrinsic causes. Only the patients with Ladd band formed from extrinsic causes CDO were fed earlier (avarage of 48 hours) and discharged earlier (avarage of 10 days) than those with intrinsic casued CDO patients.

Usually in comparison, CDO patients with intrinsic causes had lower birth weight and higher rate of prematurity than extrinsic ones. But oral feeding time and length of hospitalization were shorter then extrinsic caused CDO patients (Table 4).
Patients were discharged after tolerating oral feeding and starting to gain weight. The average length of hospitalization was $14 \pm 5$ days. Two patients with prematurity- sepsis cooccurrence were lost.

\section{Discussion}

$\mathrm{CDO}$ is the most common cause of bowel obstruction in newborns (4-7). It is seen in every 5,000 to 10,000 live births (3). It is more common in males than females, as it is in our study (5). The airdilation of the proximal duodenum and the absence of air in the distal small bowel called double-bubble image is typical on the flat and erect abdominal xray. In the prenatal period patients with polyhydramnios are pathognomonically show a presence of two fluidfilled balloons on ultrasonography (9). In addition, it may be helpful to recognize that the oral contrasting substance in the esophagus-stomach-duodenum x-ray does not migrate to the distal part of the gut (5). In our study, $24(75 \%)$ patients were diagnosed with a typical double-bubble image in flat and erect abdominal xray and $12(38 \%)$ patients were diagnosed in intrauterin period. In a study conducted by Rattan et al. (6), the diagnosis was made on the fourth day. In Kumar et al.'s study (10), the diagnosis was made on the second day. In our study, the diagnosis time was similar to Kumar et al. The most common symptomfindings in CDO are vomiting, epigastric fullness and weight loss $(6,7)$. Vomiting starts 24-38 hours after feeding begins (5). In our study, vomiting complaining was detected with a high rate $(75 \%)$. This suggests that we should think of $\mathrm{CDO}$ in differential diagnosis in newborns who have complainings of vomiting.

The conditions that cause CDO are divided into extrinsic and intrinsic ones. The most common 
Table 3. Causes of congenital duodenal obstruction

\begin{tabular}{lcc}
\hline & Number of Patients & Percent \\
\hline Atresia & 10 & 31 \\
Annular pancreas & 9 & 28 \\
Web & 7 & 22 \\
Ladd's band & 4 & 13 \\
Stenosis & 1 & 3 \\
Superior mesenteric artery & 1 & 3 \\
\hline Total & 32 & 100 \\
\hline
\end{tabular}

Table 4. Comparison of extrinsic and intrinsic causes in congenital duodenal obstruction

\begin{tabular}{lcc|cccc|}
\hline & $\begin{array}{c}\text { Female(n) } \\
\text { Percent }\end{array}$ & $\begin{array}{c}\text { Percent } \\
\text { Male(n) }\end{array}$ & $\begin{array}{c}\text { Birth } \\
\text { weight }\end{array}$ & $\begin{array}{c}\text { Length of } \\
\text { hospitalization }\end{array}$ & $\begin{array}{c}\text { Time for } \\
\text { oral feding(h) }\end{array}$ & Prematurity(n) \\
\hline intrinsic causes & $8(25 \%)$ & $10(31 \%)$ & $1850 \pm 1028$ & $13 \pm 3$ & 58 & 7 \\
extrinsic causes & $6(19 \%)$ & $8(25 \%)$ & $2010 \pm 1150$ & $15 \pm 5$ & 84 & 5 \\
\hline
\end{tabular}

extrinsic cause was found to be annulo-pancreas and the most common intrinsic cause was reported as atresia (11). Ladd's bands from extrinsic causes are mostly resected; diamond-shaped duodenoduodenostomy method is commonly used which is described in 1990 by Kimura in the treatment of all intrinsic conditions like atresia, web, stenosis and some extrinsic causes such as the annular pancreas and superior mesenteric artery pressure $(3,12)$. Depending on the location of the obstruction, duodenojejunostomy is another preferred method (6). Whether intrinsic or extrinsic causes that lead to $\mathrm{CDO}$, open surgical procedures were preferred to all of our patients. Endoscopic and laparoscopic approaches are also available. Endoscopic treatment has become popular due to the fact that abdominal incisions are not made, absence of complications such as adhesions in the abdomen and it also has an adventage of short hospitalization (11). The endoscopic approach is a good technique to confirm the presence of duodenal web which has a rare incidence of 1 in 40,000 (4,9). Endoscopically, the web can be opened by cutting it with electrocautery or laser. However, there is a risk of puncture of ampulla vater, excessive bleeding and trauma (11). In addition, the entire excision of the web is important. Because in the case of incomplete excision, the web may develop again and cause recurrent obstructions (7). Endoscopic resection may not always be successful for this reason (4). As a matter of fact, the practice of endoscopic CDO treatment has been reduced considerably because of endoscopy application certificate requirement problem for pediatric surgeons in our country in recent years.

Compared with the traditional approach, laparoscopic duodenododenostomy has advantages such as rapid recovery, early nutrition and early defecation.
However, infrastructure and experience are required for this method. The most important complication of laparoscopy is the anastomotic leak (3). It should not be forgotten that sepsis after anastomotic leak may increase mortality. In addition, all intestinal segments should be assessed in detail during surgery. Another weak side of laparoscopy is the difficulty of evaluating other atresic bowel segments and the duodenal web (3). In our study, laparoscopic method could not be performed due to lack of equipment. Furthermore, not comparing open surgery and laparoscopic method was accepted as a shortcoming of this study.

Duodenal obstructions have been reported to be associated with other congenital anomalies with a probability of $38-55 \%$ (13) The most common additional anomaly is Down Syndrome (8). Other anomalies are anorectal malformations, esophageal atresia, cardiac defects, urinary and extremity anomalies (3-6). However, these anomalies have no effect on survival (8). The most common additional anomaly in our study was Down Syndrome; no additional anomaly was found in any of the patients that we lost due to sepsis. A mortality rate of $9 \%$ (6) in a study and $22.5 \%$ in another study were reported (8). The most common cause of mortality was sepsis and cardiac pathologies (6). Two patients was lost due to sepsis in our study. These patients also had prematurity.

As a result, CDO should be considered to be a differential diagnosis especially if there is vomiting complaint in newborns with additional congenital anomalies such as Down Syndrome. Thus, time would not be lost with unnecessary medical treatment trials in this situation. Early diagnosis in CDO is essential in order to obtain satisfactory results. It should not be forgotten that delayed diagnosis,

East J Med Volume:23, Number:3, July-September/2018 
cardiac pathologies, sepsis and also prematurity are important risk factors for survival.

\section{Conflict of Interest: None}

\section{References}

1. Morris G, Kennedy A, Cochran W. Small bowel congenital anomalies: a review and update. Curr Gastroenterol Rep 2016; 18: 16.

2. Sharma S, Singh S, Sen A. Congenital double duodenal diaphragms in an infant. J Indian Assoc Pediatr Surg 2013; 18: 147-148.

3. Chiarenza SF, Bucci V, Conighi ML et al. Duodenal Atresia: Open versus MIS RepairAnalysis of Our Experience over the Last 12 Years. BioMed research international 2017; 1-8.

4. Angotti R, Molinaro F, Cobellis G, et al. Persistent Nonbilious Vomiting in a Child: Possible Duodenal Webbing. Clin Endosc 2017; 50: 191-196.

5. Eovaldi BJ, Cohen H. Duodenal Atresia And Stenosis. Statpearls 2017; 1: 5.

6. Rattan KN, Singh J, Dalal, P. Neonatal duodenal obstruction: a 15-year experience. Journal of neonatal surgery 2016; 5: 13.
7. Gupta R, Mathur P, Gubbi S, et al. More Distally Located Duodenal Webs: A Case Series. J Neonatal Surg 2016; 5: 56.

8. Erickson J, Retrouvey M, Rush J, Trace AP. Simultaneous duodenal stenosis and duodenal web in a newborn. Radiology case reports 2016; 11: 444-446.

9. Choudhry MS, Rahman N, Boyd P, Lakhoo K. Duodenal atresia: associated anomalies, prenatal diagnosis and outcome. Pediatric surgery international 2009; 25: 727-730.

10. Kumar P, Kumar C, Pandey PR, Sarin YK. Congenital Duodenal Obstruction in Neonates: Over 13 Years' Experience from a Single Centre. Journal of neonatal surgery 2016; 5(4).

11. Poddar U, Jain V, Yachha SK. Srivastava A. Congenital duodenal web: successful management with endoscopic dilatation. Endoscopy international open 2016; 4: E238-E241.

12. Aydin E. Non-classified type duodenal atresia: case report. Northern clinics of Istanbul 2015;2: 236.

13. Mustafawi AR, Hassan ME. Congenital duodenal obstruction in children: a decade's experience. Eur J Pediatr Surg 2008; 18: 93-97. 